PROCEEDINGS OF THE

AMERICAN MATHEMATICAL SOCIETY

Volume 134, Number 10, October 2006, Pages 3025-3034

S 0002-9939(06)08309-2

Article electronically published on April 13, 2006

\title{
CONVEX DOMINATES CONCAVE: AN EXCLUSION PRINCIPLE IN DISCRETE-TIME KOLMOGOROV SYSTEMS
}

\author{
RYUSUKE KON \\ (Communicated by Carmen C. Chicone)
}

\begin{abstract}
We establish an exclusion principle in discrete-time Kolmogorov systems by using average Liapunov functions. The exclusion principle shows that a weakly dominant species with a convex logarithmic growth rate function eliminates species with concave logarithmic growth rate functions. A general result is applied to specific population models. This application gives an improved exclusion principle for the specific population models.
\end{abstract}

\section{INTRODUCTION}

In this paper, we study population models governed by difference equations. One of the most popular types of such population models has the following form:

$$
x_{i}(t+1)=x_{i}(t) g_{i}(x(t)), \quad i=1, \ldots, n .
$$

This type of population model is called Kolmogorov type. The valuable $x_{i}(t)$ represents a population density of species $i$ at time $t$, and $x(t)$ is a vector of population densities $x(t)=\left(x_{1}(t), \ldots, x_{n}(t)\right)^{\top}$. We focus on the solutions of (1.1) in the nonnegative cone $\mathbb{R}_{+}^{n}:=\left\{x \in \mathbb{R}^{n}: x_{1} \geq 0, \ldots, x_{n} \geq 0\right\}$. The function $g_{i}$ is a growth rate of species $i$, which depends on the vector of population densities $x(t)$. Depending on the properties of the functions $g_{i}$, system (1.1) can represent several kinds of species interactions, e.g., competitive, cooperative and predator-prey interactions (if $g_{i}$ and $g_{j}$ are decreasing (resp. increasing) functions in $x_{j}$ and $x_{i}$, respectively, then species $i$ and $j$ are in competition (resp. cooperation) with each other, and if $g_{i}$ is increasing in $x_{j}$ and $g_{j}$ is decreasing in $x_{i}$, then the interaction between species $i$ and $j$ is a predator-prey (species $i$ is a predator and $j$ is a prey)).

From several points of view, the dynamics of system (1.1) have been investigated. For example, in [9, 11, 13, 14, 15, criteria which ensure species coexistence in the sense of permanence are studied. In contrast with these studies, Franke and Yakubu [4, 5, 6, 7] obtained several criteria which ensure that some species is dominant in the system, i.e., the species eliminates other species from the system irrespective of the initial population densities. More precisely, dominance is defined as follows.

Received by the editors March 1, 2005 and, in revised form, April 26, 2005.

2000 Mathematics Subject Classification. Primary 92B05; Secondary 39A11.

Key words and phrases. Exclusion principle, dominance, average Liapunov functions.

The author was supported by the 21st Century COE Program "Development of Dynamic Mathematics with High Functionality (Kyushu University)" of the Ministry of Education, Culture, Sports, Science and Technology of Japan.

(C)2006 American Mathematical Society 3025

Reverts to public domain 28 years from publication 
Definition 1.1. The species $k$ is said to be dominant if $\lim _{t \rightarrow \infty} x_{i}(t)=0, i \in$ $\{1, \ldots, n\} \backslash k$, for every $x(0) \in \mathbb{R}_{+}^{n}$ with $x_{k}(0)>0$.

We call the criteria which ensure dominance of some species an exclusion principle. From the definition of dominance, it is clear that coexistence of $n$ species in system (1.1) is impossible if there is a dominant species. Therefore, non-existence of dominant species is necessary for coexistence of $n$ species in system (1.1). From the point of view of evolutionary biology, an exclusion principle is also important to evaluate the possibility that a successfully invading mutant species replaces a resident species (e.g., see Geritz et al. [8]).

The purpose of this paper is to provide a new result on dominance in system (1.1), which is not only deduced from the earlier results [4, 6, 7]. In the studies of dominance, the following concept of weak dominance is important:

Definition 1.2. The species $k$ is said to be weakly dominant if the two subsets $D_{k}^{-}$ and $\bigcup_{i \in\{1, \ldots, n\} \backslash k} D_{i}^{+}$of $\mathbb{R}_{+}^{n}$ are disjoint, where $D_{i}^{+}:=\left\{x \in \mathbb{R}_{+}^{n}: g_{i}(x) \geq 1\right\}$ and $D_{i}^{-}:=\left\{x \in \mathbb{R}_{+}^{n}: g_{i}(x) \leq 1\right\}$.

From the definition of $D_{i}^{+}$and $D_{i}^{-}$, the population density of species $i$ is nondecreasing and non-increasing in $D_{i}^{+}$and $D_{i}^{-}$, respectively. The intersection $D_{i}^{+} \cap$ $D_{i}^{-}$represents a null cline in the sense that $x_{i}(t+1)-x_{i}(t)$ is zero if $x(t) \in D_{i}^{+} \cap D_{i}^{-}$. Thus, on the null cline $D_{i}^{+} \cap D_{i}^{-}$, the population density of species $i$ remains constant in one unit of time. A point $x \in \bigcap_{i=1}^{n}\left(D_{i}^{+} \cap D_{i}^{-}\right)$is a candidate for a positive fixed point, which is a fixed point in the interior of $\mathbb{R}_{+}^{n}$. Therefore, if a weakly dominant species exists, system (1.1) does not possess a positive fixed point. This implies that $n$ species cannot coexist at a fixed point. It is known that if all growth rate functions $g_{i}$ have exponential form, then weak dominance implies dominance (see [1, [5]). However, it is also known that, in general, weak dominance does not always imply dominance. For example, in [4, 5, 6, 7, 17, it is shown, by using specific examples of (1.1) with $n=2$, that stable periodic solutions can exist in the interior of $\mathbb{R}_{+}^{2}$ even if there is a weakly dominant species. Moreover, coexistence with chaotic oscillation is also possible under the assumption of weak dominance (see [16, 17]). These facts lead to an interesting problem of finding an additional condition that ensures that weak dominance implies dominance.

After the next preliminary section, we consider this problem and obtain such an additional assumption (Theorem 3.2). Our result shows that if the logarithmic growth rate function $\ln g_{k}$ is convex and all other functions $\ln g_{i}, i \in\{1, \ldots, n\} \backslash k$, are concave, then weak dominance of species $k$ implies its dominance. In Section 4 , we apply our result to specific population models, in which each growth rate function $g_{i}$ is a function of the weighted total population density $\sum_{j=1}^{n} a_{i j} x_{j}(t)$. The final section discusses the differences between our result and the earlier ones by Franke and Yakubu [4, 6, 7. We will see that there is no inclusion relation between them. But, we will also see that our result has an advantage in its application to specific models.

\section{Preliminaries}

In this section, we introduce some notations and theorems, which are used in the following sections.

Let $(X, d)$ be a metric space with metric $d$. A map $f: X \rightarrow X$ defines a discrete semi-dynamical system $\pi: \mathbb{Z}_{+} \times X \rightarrow X$ by $\pi(t, x)=f^{t}(x)$, where $\mathbb{Z}_{+}:=$ 
$\{0,1,2, \ldots\}$ and $f^{t}(x)$ denotes the $t$-th iterate of $x$ under $f$. Throughout this section, we assume that $f: X \rightarrow X$ is continuous. Let $\omega(x)$ be the omega limit set of $x$ and let $\gamma^{+}(x)$ be the semi-orbit through $x$, i.e., $\omega(x):=\left\{y \in X: f^{t_{j}}(x) \rightarrow\right.$ $y$ for some subsequence $\left.t_{j} \rightarrow \infty\right\}$ and $\gamma^{+}(x):=\left\{y \in X: y=f^{t}(x)\right.$ for $\left.t \in \mathbb{Z}_{+}\right\}$. For a subset $N$ of $X$, we define $\omega(N):=\bigcup_{x \in N} \omega(x)$ and $\gamma^{+}(N):=\bigcup_{x \in N} \gamma^{+}(x)$. N is said to be forward invariant if $f(N) \subset N$. For subsets $N$ and $Y$ of $X, N$ is said to be absorbing for $Y$ if it is forward invariant and $\gamma^{+}(x) \cap N \neq \emptyset$ for every $x \in Y$.

The following lemma is used to construct a compact absorbing set of system (1.1) in Section 4 (see also Hofbauer et al. 9], Lemma 2.1).

Lemma 2.1 (Hutson [12, Lemma 2.1). Let $Y \subset X$ be open, and let $N$ be open with a compact closure $\bar{N} \subset Y$. Assume that $Y$ is forward invariant and that $\gamma^{+}(x) \cap N \neq \emptyset$ for every $x \in Y$. Then $M=\gamma^{+}(\bar{N})$ is a compact absorbing set for $Y$.

Now we introduce theorems of average Liapunov functions. In the next section, the following two theorems are used to show that the extinction state of some species is repelling or attracting, respectively.

Theorem 2.2 (Hutson [12, Theorem 2.2). Assume that $X$ is compact and that $S$ is a compact subset of $X$ with empty interior. Suppose that there is a continuous function $P: X \rightarrow \mathbb{R}_{+}$which satisfies the following conditions:

(a) $P(x)=0 \Longleftrightarrow x \in S$,

(b) $\sup _{\substack{T \geq 0 \\ T \geq 0 \rightarrow x \\ y \in X}} \frac{P\left(f^{T}(y)\right)}{P(y)}>1 \quad(x \in S)$.

Then there is a compact absorbing set $M$ for $X \backslash S$ with $M \cap S=\emptyset$.

Theorem 2.3 (Kon and Takeuchi [14, Lemma 14). Let $X$ and $S$ be the same as those in Theorem 2.2. Suppose that there is a continuous function $P: X \rightarrow \mathbb{R}_{+}$ which satisfies the following conditions:

(a) $P(x)=0 \Longleftrightarrow x \in S$,

(b) $\inf _{T \geq 0} \limsup _{\substack{y \rightarrow x \\ y \in X \backslash S}} \frac{P\left(f^{T}(y)\right)}{P(y)}<1 \quad(x \in S)$,

(c) $\inf _{T \geq 0} \frac{P\left(f^{T}(x)\right)}{P(x)}<1 \quad(x \in X \backslash S)$.

Then $\omega(X) \subset S$, i.e., all solutions in $X$ converge to $S$ as $t \rightarrow \infty$.

\section{MAin Results}

In this section, we obtain the main theorem (Theorem 3.2) of exclusion principles for system (1.1) by using the theorems in the pervious section.

Since system (1.1) represents population dynamics, we are only interested in its orbits restricted in the non-negative cone $\mathbb{R}_{+}^{n}$. In order that population densities do not become negative and species do not go extinct in finite time, we introduce the following assumption:

$(\mathrm{H}): g_{i}: \mathbb{R}_{+}^{n} \rightarrow \mathbb{R}_{+}$is positive and continuous for each $i=1, \ldots, n$.

We see that if assumption $(\mathrm{H})$ holds, then both $\mathbb{R}_{+}^{n}$ and its interior, int $\mathbb{R}_{+}^{n}$, are forward invariant under system (1.1), and the map $f: \mathbb{R}_{+}^{n} \rightarrow \mathbb{R}_{+}^{n}$ defined by $f=\left(x_{1} g_{1}, \ldots, x_{n} g_{n}\right)^{\top}$ is continuous. 
Define $S_{i}:=\left\{x \in \mathbb{R}_{+}^{n}: x_{i}=0\right\}$, which is the set of the state where species $i$ is extinct. Denote $\bigcap_{i=1}^{n} S_{i}$ by $O$, which is the set consisting only of the origin. From the equation form of system (1.1), it is clear that each $S_{i}$ is forward invariant. Therefore, every union and every intersection of $S_{i}$ is also forward invariant.

We define the limit set of time average of solutions as follows (see [10]):

$$
\mu(x):=\left\{y \in \mathbb{R}_{+}^{n}: \lim _{j \rightarrow \infty} \frac{1}{t_{j}} \sum_{t=0}^{t_{j}-1} x(t)=y \text { for some sequence } t_{j} \rightarrow \infty\right\},
$$

where $x(t)$ is a solution of system (1.1) with $x(0)=x$. In the following lemma, we obtain the conditions that $\mu(x)$ must satisfy under the assumption that $\ln g_{i}$ is convex or concave:

Lemma 3.1. Let $M \subset \mathbb{R}_{+}^{n}$ be convex, and let $X \subset \mathbb{R}_{+}^{n}$ be compact with $X \subset M \subset$ $\mathbb{R}_{+}^{n}$. Assume that $(\mathrm{H})$ holds. Suppose that $X$ is forward invariant under (1.1). If $\omega(x) \cap\left(X \backslash S_{i}\right) \neq \emptyset$ for some $x \in X$, then

(i) $\mu(x) \cap D_{i}^{+} \neq \emptyset$ if $\ln g_{i}$ is concave on $M$,

(ii) $\mu(x) \cap D_{i}^{-} \neq \emptyset$ if $\ln g_{i}$ is convex on $M$.

Proof. Let $x(t)=\left(x_{1}(t), \ldots, x_{n}(t)\right)$ be a solution of (1.1) with $x(0)=x$. From (1.1), we have

$$
\begin{aligned}
\frac{x_{i}(T)}{x_{i}(0)}=\frac{x_{i}(T)}{x_{i}(T-1)} \frac{x_{i}(T-1)}{x_{i}(T-2)} \ldots \frac{x_{i}(1)}{x_{i}(0)} & =\prod_{t=0}^{T-1} g_{i}(x(t)), \\
\frac{\ln x_{i}(T)-\ln x_{i}(0)}{T} & =\frac{1}{T} \sum_{t=0}^{T-1} \ln g_{i}(x(t)) .
\end{aligned}
$$

The assumption $\omega(x) \cap\left(X \backslash S_{i}\right) \neq \emptyset$ implies that there exist a sequence $T_{j} \rightarrow \infty$ and a $\delta>0$ such that $x_{i}\left(T_{j}\right) \geq \delta$ for all $j \in \mathbb{Z}_{+}$. By the compactness of $X$ and the existence of $\delta$, we have

$$
0=\lim _{j \rightarrow \infty} \frac{1}{T_{j}} \sum_{t=0}^{T_{j}-1} \ln g_{i}(x(t))
$$

For the case (i), we can apply Jensen's inequality to the concave function $\ln g_{i}$ as follows:

$$
\frac{1}{T_{j}} \sum_{t=0}^{T_{j}-1} \ln g_{i}(x(t)) \leq \ln g_{i}\left(\frac{1}{T_{j}} \sum_{t=0}^{T_{j}-1} x(t)\right)
$$

Then there exists a subsequence, again denoted by $T_{j} \rightarrow \infty$, such that

$$
0 \leq \ln g_{i}\left(\lim _{j \rightarrow \infty} \frac{1}{T_{j}} \sum_{t=0}^{T_{j}-1} x(t)\right)
$$

This implies that $\mu(x) \cap D_{i}^{+} \neq \emptyset$. Similarly, we can prove case (ii).

The following theorem is the main theorem of this paper, and it gives testable exclusion principles for specific population models (see the next section).

Theorem 3.2. Let $M$ and $X$ be the same as those in Lemma 3.1, and let $X_{i}^{+}=$ $D_{i}^{+} \cap X$ and $X_{i}^{-}=D_{i}^{-} \cap X$. Assume that $(\mathrm{H})$ holds and $X \cap O=\emptyset$. Suppose that the function $\ln g_{k}$ is convex and the functions $\ln g_{i}, i \in\{1, \ldots, n\} \backslash k$, are concave on $M$. If $X_{k}^{-}$and $\bigcup_{i \in\{1, \ldots, n\} \backslash k} X_{i}^{+}$are disjoint, then $\omega\left(X \backslash S_{k}\right) \subset\left(\bigcap_{i \in\{1, \ldots, n\} \backslash k} S_{i}\right) \backslash S_{k}$. 
Proof. Without loss of generality, we assume $k=1$. By using Theorem 2.2, we shall show that there exists a compact absorbing set $X^{\prime} \subset X \backslash S_{1}$ for $X \backslash S_{1}$. Define $P_{1}: X \rightarrow \mathbb{R}_{+}$as $P_{1}(x)=x_{1}$. It is clear that $P_{1}(x)=0$ if and only if $x \in S_{1} \cap X$, i.e., condition (a) of Theorem 2.2 holds. Let us check condition (b) of Theorem 2.2. For every $x \in S_{1} \cap X$ we have

$$
\sigma_{1}(x)=\sup _{T \geq 0} \liminf _{\substack{y \rightarrow x \\ y \in X \backslash S_{1}}} \frac{P_{1}\left(f^{T}(y)\right)}{P_{1}(y)}=\sup _{T \geq 0} \liminf _{\substack{y \rightarrow x \\ y \in X \backslash S_{1}}} \frac{y_{1}(T)}{y_{1}(T-1)} \cdots \frac{y_{1}(2)}{y_{1}(1)} \frac{y_{1}(1)}{y_{1}(0)},
$$

where $y(t)=\left(y_{1}(t), \ldots, y_{n}(t)\right)$ is a solution of (1.1) with $y(0)=y$. By using the continuity of $f$, we have

$$
\sigma_{1}(x)=\sup _{T \geq 0} \prod_{t=0}^{T-1} g_{1}(x(t))=\sup _{T \geq 0}\left(\exp \left[\frac{1}{T} \sum_{t=0}^{T-1} \ln g_{1}(x(t))\right]\right)^{T},
$$

where $x(t)$ is a solution of (1.1) with $x(0)=x$. Since the function $\ln g_{1}$ is convex on $M$, Jensen's inequality implies

$$
\frac{1}{T} \sum_{t=0}^{T-1} \ln g_{1}(x(t)) \geq \ln g_{1}\left(\frac{1}{T} \sum_{t=0}^{T-1} x(t)\right) .
$$

Since $X \cap O=\emptyset$, for every $x \in S_{1} \cap X$ there exists an $i \in\{2, \ldots, n\}$ such that $\omega(x) \cap\left(X \backslash S_{i}\right) \neq \emptyset$. Then it follows from Lemma 3.1 that for every $x \in S_{1} \cap X$ there exists an $i \in\{2, \ldots, n\}$ such that $\mu(x) \cap D_{i}^{+} \neq \emptyset$ holds. By the assumption $X_{1}^{-} \cap\left(\bigcup_{i=2}^{n} X_{i}^{+}\right)=\emptyset$, we see that for every $x \in S_{1} \cap X$ there exists a sequence $T_{j} \rightarrow \infty$ such that $\ln g_{1}\left(\sum_{t=0}^{T_{j}-1} x(t) / T_{j}\right)>0$ for a sufficiently large $j \in \mathbb{Z}_{+}$. This implies that $\sigma_{1}(x)>1$ for every $x \in S_{1} \cap X$. Hence, by Theorem 2.2, we see that there exists a compact absorbing set $X^{\prime} \subset X \backslash S_{1}$ for $X \backslash S_{1}$.

By using Theorem 2.3, we shall show that every solution with the initial condition $x \in X^{\prime}$ converges to the intersection of $S_{i}^{\prime}:=S_{i} \cap X^{\prime}, i=2, \ldots, n$, i.e., $\omega\left(X^{\prime}\right) \subset$ $\bigcap_{i=2}^{n} S_{i}^{\prime}$. Let $m$ be an arbitrary number in $\{2, \ldots, n\}$, and define $P_{m}: X^{\prime} \rightarrow \mathbb{R}_{+}$as $P_{m}(x)=x_{m}$ and

$$
\sigma_{m}(x)= \begin{cases}\inf _{T \geq 0} \limsup _{\substack{y \rightarrow x \\ y \in X^{\prime} \backslash S_{m}^{\prime}}} \frac{P_{m}\left(f^{T}(y)\right)}{P_{m}(y)} & \text { if } x \in S_{m}^{\prime}, \\ \inf _{T \geq 0} \frac{P_{m}\left(f^{T}(x)\right)}{P_{m}(x)} & \text { if } x \in X^{\prime} \backslash S_{m}^{\prime} .\end{cases}
$$

Then it is clear that $P_{m}(x)=0$ if and only if $x \in S_{m}^{\prime}$, i.e., condition (a) of Theorem 2.3 is satisfied. For every $x \in X^{\prime}$ we have

$$
\sigma_{m}(x)=\inf _{T \geq 0} \prod_{t=0}^{T-1} g_{m}(x(t))=\inf _{T \geq 0}\left(\exp \left[\left(\frac{1}{T} \sum_{t=0}^{T-1} \ln g_{m}(x(t))\right)\right]\right)^{T},
$$

where the continuity of the function $f$ is used and $x(t)$ is a solution of (1.1) with $x(0)=x$. Since the function $\ln g_{m}$ is concave on $M$, Jensen's inequality implies

$$
\frac{1}{T} \sum_{t=0}^{T-1} \ln g_{m}(x(t)) \leq \ln g_{m}\left(\frac{1}{T} \sum_{t=0}^{T-1} x(t)\right)
$$

Since $X^{\prime}$ is a compact invariant set with $X^{\prime} \cap S_{1}=\emptyset$, it is clear that $\omega(x) \cap\left(X^{\prime} \backslash S_{1}\right) \neq$ $\emptyset$ for every $x \in X^{\prime}$. Then, by Lemma 3.1, we see that $\mu(x) \cap D_{1}^{-} \neq \emptyset$ holds for 
every $x \in X^{\prime}$. Therefore, the assumption $X_{1}^{-} \cap\left(\bigcup_{i=2}^{n} X_{i}^{+}\right)=\emptyset$ implies that for every $x \in X^{\prime}$ there exists a sequence $T_{j} \rightarrow \infty$ such that $\ln g_{m}\left(\sum_{t=0}^{T_{j}-1} x(t) / T_{j}\right)<0$ for a sufficiently large $j \in \mathbb{Z}_{+}$. This implies that $\sigma_{m}(x)<1$ for every $x \in X^{\prime}$. By Theorem 2.3 , we see that $\omega\left(X^{\prime}\right) \subset S_{m}^{\prime}$. This completes the proof since $m$ is an arbitrary number in $\{2, \ldots, n\}$.

Remark 3.3. If $\ln g_{k}$ is convex and $\ln g_{i}, i \in\{1, \ldots, n\} \backslash k$ are concave on $\mathbb{R}_{+}^{n}$, and (1.1) has a compact absorbing set $X$ for $\mathbb{R}_{+}^{n} \backslash O$ satisfying $X \cap O=\emptyset$, then weak dominance of species $k$ implies its dominance.

\section{Applications}

In this section, we apply our main theorem (Theorem [3.2) to the following system:

$$
x_{i}(t+1)=x_{i}(t) h_{i}\left(\sum_{j=1}^{n} a_{i j} x_{j}(t)\right), \quad i=1, \ldots, n,
$$

where $a_{i j}>0, i, j \in\{1, \ldots, n\}$. We assume that each $h_{i}: \mathbb{R}_{+} \rightarrow \mathbb{R}_{+}$satisfies the following conditions:

(A1): $h_{i}$ is positive and continuous,

(A2): $h_{i}$ is strictly decreasing, and $h_{i}\left(x_{i}^{*}\right)=1$ at some $x_{i}^{*}>0$.

Note that (A2) implies that $h_{i}(0)>1$ for every $i \in\{1, \ldots, n\}$. Since the function $h_{i}$ is a function of the weighted total population density, the null cline $D_{i}^{+} \cap D_{i}^{-}$is the simplex $\sum_{j=1}^{n} a_{i j} x_{j}=x_{i}^{*}$. We define $X_{i j}=x_{i}^{*} / a_{i j}$.

Under the assumptions, by using Lemma 2.1, we can construct a compact absorbing set $X$ for $\mathbb{R}_{+}^{n} \backslash O$ satisfying $X \cap O=\emptyset$ as follows.

Lemma 4.1. If (A1) and (A2) hold, then system (4.1) has a compact absorbing set $X$ for $\mathbb{R}_{+}^{n} \backslash O$ satisfying $X \cap O=\emptyset$.

Proof. By (A1), the non-negative cone $\mathbb{R}_{+}^{n}$ is forward invariant. By (A2), there exists an $L>0$ such that $p \geq L$ implies $h_{i}(p)<1$ for all $i \in\{1, \ldots, n\}$. Let $N:=\left\{x \in \mathbb{R}_{+}^{n}:\left(\min _{i, j \in\{1, \ldots, n\}} a_{i j}\right)\left(x_{1}+\cdots+x_{n}\right)<L\right\} . N$ is an open subset of $\mathbb{R}_{+}^{n}$ with a compact closure $\bar{N} \subset \mathbb{R}_{+}^{n}$. Let $x(t)$ be a solution of (4.1) with $x(0) \in \mathbb{R}_{+}^{n}$. If $x(t) \in \mathbb{R}_{+}^{n} \backslash N$, then $x_{i}(t+1)<x_{i}(t)$ holds for all $i \in\{1, \ldots, n\}$. Suppose that $x(t) \in \mathbb{R}_{+}^{n} \backslash N$ for all $t \geq 0$. Since $h_{i}$ is strictly decreasing, there exists a $\delta \in(0,1)$ such that $\max _{i \in\{1, \ldots, n\}} h_{i}(p) \leq \delta$ for all $p \geq L$. Then, by (4.1), we have

$$
\begin{aligned}
\sum_{i=1}^{n} x_{i}(t) & =\sum_{i=1}^{n} x_{i}(t-1) h_{i}\left(\sum_{j=1}^{n} a_{i j} x_{j}(t-1)\right) \\
& \leq \delta^{t} \sum_{i=1}^{n} x_{i}(0) .
\end{aligned}
$$

Hence, $\sum_{i=1}^{n} x_{i}(t) \rightarrow 0$ as $t \rightarrow \infty$. This is a contradiction. Therefore, for every $x(0) \in \mathbb{R}_{+}^{n}$ there exists a $t \geq 0$ such that $x(t) \in N$, i.e., $\gamma^{+}(x) \cap N \neq \emptyset$ for every $x \in \mathbb{R}_{+}^{n}$. By Lemma 2.1, there exists a compact absorbing set $M$ for $\mathbb{R}_{+}^{n}$.

By using Lemma 2.1, we can also construct a compact absorbing set $X$ for $M \backslash O$ satisfying $X \cap O=\emptyset$ as follows. By (A2), there exists an $l>0$ such that $0 \leq p \leq l$ implies $h_{i}(p)>1$ for all $i \in\{1, \ldots, n\}$. Then, by the same argument used above, we can show that there exists an open subset $V$ of $M$ with compact closure $\bar{V} \subset M \backslash O$ 
such that $\gamma^{+}(x) \cap V \neq \emptyset$ for every $M \backslash O$. (Note that $V_{l}:=\left\{x \in \mathbb{R}_{+}^{n}: x_{1}+\cdots+x_{n}>\right.$ $l\}$ is an open subset of $\mathbb{R}_{+}^{n}$, and $V_{l} \cap M \neq \emptyset$ holds for all small $l>0$ since $M$ is an absorbing set for $\mathbb{R}_{+}^{n}$ and $\mathbb{R}_{+}^{n} \backslash O$ is forward invariant. This implies that $V_{l} \cap M$ is an open subset of $M$ for all small $l>0$.) Hence $X:=\gamma^{+}(\bar{V})$ is a compact absorbing set for $\mathbb{R}_{+}^{n} \backslash O$ satisfying $X \cap O=\emptyset$.

Theorem 3.2 with Lemma 4.1 leads to the following corollary.

Corollary 4.2. Consider system (4.1) with (A1) and (A2). Assume that the function $\ln h_{k}$ is convex and the functions $\ln h_{i}, i \in\{1, \ldots, n\} \backslash k$, are concave on $\mathbb{R}_{+}$. Then species $k$ is dominant if $X_{i 1}<X_{k 1}, \ldots, X_{i n}<X_{k n}$ for all $i \in\{1, \ldots, n\} \backslash k$.

Proof. Each $D_{i}^{+} \cap D_{i}^{-}$is the simplex $a_{i 1} x_{1}+\cdots+a_{i n} x_{n}=x_{i}^{*}$. Then the condition that $X_{i 1}<X_{k 1}, \ldots, X_{i n}<X_{k n}$ for all $i \in\{1, \ldots, n\} \backslash k$ implies $\left(\bigcup_{i \in\{1, \ldots, n\} \backslash k} D_{i}^{+}\right) \cap$ $D_{k}^{-}=\emptyset$. Furthermore, the convexity and concavity of $\ln h_{i}$ on $\mathbb{R}_{+}$imply the convexity and concavity of $\ln g_{i}\left(x_{1}, \ldots, x_{n}\right)=\ln h_{i}\left(\sum_{i=1}^{n} a_{i j} x_{j}\right)$ on $\mathbb{R}_{+}^{n}$, respectively. Hence, Theorem 3.2 with Lemma 4.1 completes the proof.

Remark 4.3. If $a_{i 1}=\cdots=a_{i n}$ holds for all $i \in\{1, \ldots, n\}$, then the condition that $X_{i 1}<X_{k 1}, \ldots, X_{i n}<X_{k n}$ for all $i \in\{1, \ldots, n\} \backslash k$ is reduced to the condition that $X_{i i}<X_{k k}$ for all $i \in\{1, \ldots, n\} \backslash k$. Furthermore, this condition becomes necessary and sufficient for dominance of species $k$. Indeed, in this case, a segment connecting the fixed points on the $x_{k}$ - and $x_{i}$-axes becomes a continuum of fixed points if $X_{i i}=X_{k k}$, and the fixed point on the $x_{i}$-axis attracts some orbits on the interior of the $x_{k}-x_{i}$ face if $X_{i i}>X_{k k}$.

The following systems are specific examples of system (4.1):

$$
\begin{gathered}
\left\{\begin{aligned}
x_{1}(t+1) & =x_{1}(t) \frac{\lambda}{\left(x_{1}(t)+\alpha x_{2}(t)+\beta\right)^{\gamma},} \\
x_{2}(t+1) & =x_{2}(t) \exp \left\{r-a\left(b x_{1}(t)+x_{2}(t)\right)\right\},
\end{aligned}\right. \\
\left\{\begin{array}{l}
x_{1}(t+1)=x_{1}(t)\left[\exp \left\{r_{1}-a_{1}\left(x_{1}(t)+x_{2}(t)\right)\right\}+s\right], \\
x_{2}(t+1)=x_{2}(t) \exp \left\{r_{2}-a_{2}\left(x_{1}(t)+x_{2}(t)\right)\right\},
\end{array}\right.
\end{gathered}
$$

where the parameters are positive and satisfy $\lambda / \beta^{\gamma}>1$ and $0 \leq s<1$. We can find the studies of (4.2) and (4.3) in [5, 6] and [2, 16, 17], respectively. It is clear that both (4.2) and (4.3) satisfy the assumptions (A1) and (A2). It is straightforward to confirm that the following functions $\ln h_{1}$ and $\ln \bar{h}_{1}$ are convex and $\ln h_{2}$ and $\ln \bar{h}_{2}$ are concave (and convex):

$$
\begin{aligned}
& \ln h_{1}(p)=\ln \left\{\lambda /(p+\beta)^{\gamma}\right\}, \quad \ln h_{2}(p)=r-p, \\
& \ln \bar{h}_{1}(p)=\ln \left\{\exp \left(r_{1}-p\right)+s\right\}, \quad \ln \bar{h}_{2}=r_{2}-p .
\end{aligned}
$$

Hence, the following two corollaries are immediate consequences of Corollary 4.2 and its remark.

Corollary 4.4. Species 1 of (4.2) is dominant if

$$
\lambda^{\frac{1}{\gamma}}-\beta>\frac{r}{a b} \text { and } \frac{\lambda^{\frac{1}{\gamma}}-\beta}{\alpha}>\frac{r}{a} \text {. }
$$

Corollary 4.5. Species 1 of (4.3) is dominant if and only if

$$
\frac{r_{1}-\ln (1-s)}{a_{1}}>\frac{r_{2}}{a_{2}} \text {. }
$$


Note that Corollary 4.4 is identical to Theorem 5.2 of Franke and Yakubu [5], which was obtained by different methods, and Corollary 4.5)improves Theorem 5 of Yakubu [17, in which an exclusion principle was obtained to consider the possibility that the endangered species $x_{1}$ could be saved by its planting. It is known that species 2 does not always eliminate species 1 in systems (4.2) and (4.3) even if species 2 is weakly dominant (see [5] and [17]). This shows that, in general, a weakly dominant species $k$ with concave $\ln g_{k}$ does not always eliminate species $j$ with convex $\ln g_{j}$.

\section{Discussion}

This paper considered the dynamics of system (1.1), and gave a checkable condition under which weak dominance implies dominance, that is, exclusion of all weakly dominated species irrespective of initial population densities. This result was applied to the competition model (4.1), including the models (4.2) and (4.3) as a special case. In this application, we obtained sufficient conditions that ensure exclusion of the weakly dominated species in (4.2) and (4.3). One of the sufficient conditions reconfirmed the previous result by Franke and Yakubu [5], and the other one improved the result by Yakubu [17].

In the rest of this section, we discuss the differences between our results and the earlier ones by Franke and Yakubu [4, 6, 7. To simplify the discussion, we consider system (4.1) with $n=2$ and $a_{i j}=1, i, j \in\{1,2\}$ and assume that (A1) and (A2) hold. The earlier results by Franke and Yakubu [4, 6, 7, involve three kinds of methods, which are described as the following theorems for the simplified system:

Theorem 5.1 (cf. Theorem 3.1 of [7]). If $\ln h_{1}(p)>\ln h_{2}(p)$ for all $p \in \mathbb{R}_{+}$, then species 1 is dominant.

Theorem 5.2 (cf. Theorem 4.2 of [7]). Suppose that $\mathbb{R}_{+}^{2} \backslash D_{1}^{-}$has no return points, i.e., for every $x \in \mathbb{R}_{+}^{2} \backslash D_{1}^{-}$there are no positive integers $m$ and $n, m>n$, such that $f^{n}(x) \notin \mathbb{R}_{+}^{2} \backslash D_{1}^{-}$but $f^{m}(x) \in \mathbb{R}_{+}^{2} \backslash D_{1}^{-}$. Then species 1 is dominant if it is weakly dominant.

Theorem 5.3 (Theorem 6.1 of [4]). Let $T_{i}, i=1,2$, be a forward invariant closed interval which attracts all orbits on the $x_{i}$-axis with $x_{i}(0)>0$. If $\max T_{2}<\min T_{1}$, then species 1 is dominant.

In order to consider an advantage of our results, we shall show, by using numerical investigations, that the above three theorems cannot provide our result obtained in Corollary 4.5. Figure 1 shows the graph of the functions $\ln \bar{h}_{i}, i=1,2$, of system (4.3) for a parameter set that satisfies the condition of Corollary 4.5. From this figure, we see that Theorem 5.1 is not applicable to this case since $\ln \bar{h}_{1}(p)>\ln \bar{h}_{2}(p)$ is invalid at some $p>0$. Figure2(a) depicts a positive solution of system (4.3) with the same parameters as in Figure 1. The positive solution with an initial point in $D_{1}^{-}$leaves there and enters $\mathbb{R}_{+}^{2} \backslash D_{1}^{-}$, and it converges to a 2 -cycle on the $x_{1}$-axis while oscillating between $\mathbb{R}_{+}^{2} \backslash D_{1}^{-}$and $D_{1}^{-}$. Hence, it is clear that $\mathbb{R}_{+}^{2} \backslash D_{1}^{-}$has a return point. This implies that Theorem 5.2 is not applicable to this case. Figure 2(b) illustrates the boundary dynamics of system (4.3) with the same parameters as in Figure 1. The solution on the $x_{1}$-axis converges to a 2 -cycle, while the one on the $x_{2}$-axis oscillates with large amplitude. Since $T_{i}$ should include all omega limit 


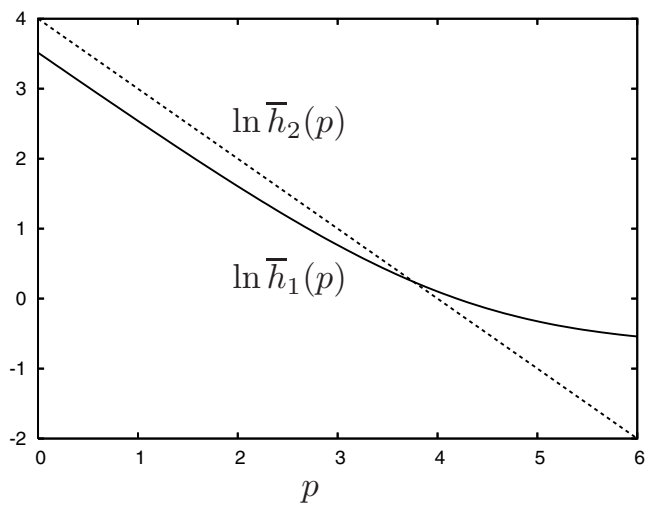

Figure 1. The graphs of the functions $\ln \bar{h}_{1}(p)$ and $\ln \bar{h}_{2}(p)$. The parameters are $r_{1}=3.5, r_{2}=4, a_{1}=1, a_{2}=1, s_{1}=0.5$.
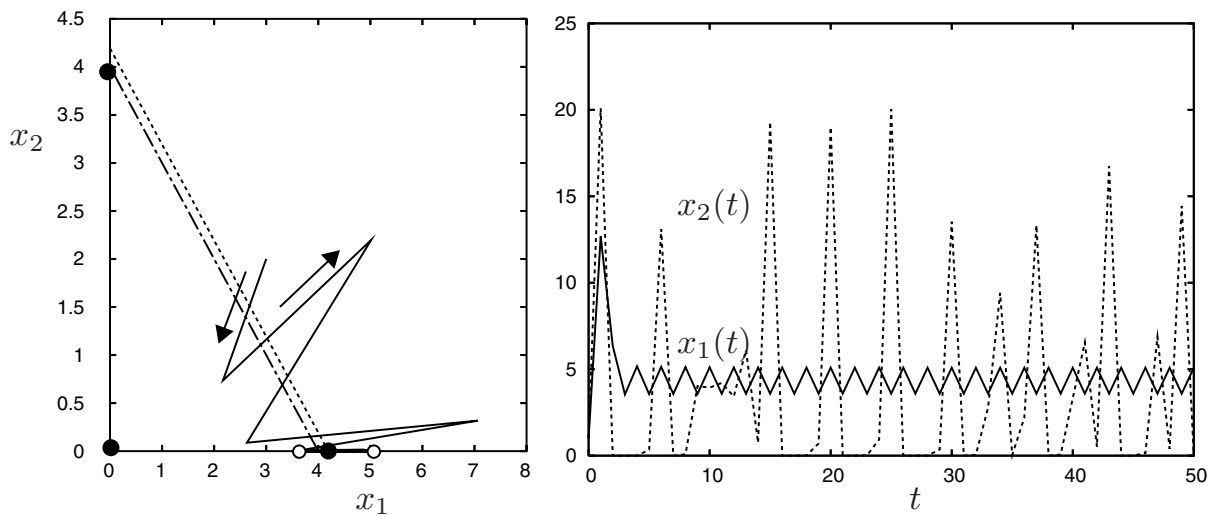

Figure 2. (a) The phase plane of System (4.3). The solid line represents a solution of System (4.3) with $\left(x_{1}(0), x_{2}(0)\right)=(3,2)$. The dashed and dot-dashed lines represent the null clines $D_{1}^{+} \cap D_{1}^{-}$ and $D_{2}^{+} \cap D_{2}^{-}$, respectively. The black dots represent fixed points and the white ones on the $x_{1}$-axis represent a 2-cycle. (b) The dynamics of System (4.3) on the $x_{i}$-axis. The solid and dashed lines represent solutions on the $x_{1}$ - and $x_{2}$-axes, respectively. The parameters are $r_{1}=3.5, r_{2}=4, a_{1}=1, a_{2}=1, s_{1}=0.5$.

sets of boundary orbits with $x_{i}(0)>0$, Figure 2(b) implies that $\max T_{2}<\min T_{1}$ does not hold. Therefore, Theorem 5.3 is also not applicable to this case.

Although Theorems 5.155.3 cannot prove Corollary 4.5. we see that these three theorems have advantages. That is, the theorems cannot be deduced from our result (Theorem 3.2). In fact, it is easy to construct a specific model whose growth rate functions satisfy $\ln \bar{h}_{1}(p)>\ln \bar{h}_{2}(p)$ for all $p \in \mathbb{R}_{+}$but have neither convexity nor concavity. Moreover, we can find specific models to which we cannot apply our results but can apply Theorems 5.2 or 5.3 . For example, see Theorem 5.2 of $[6]$ and Theorem 1 of [3]. In Theorem 5.2 of [6] and Theorem 1 of [3], dominance of 
species 2 in systems (4.2) and (4.3) have been investigated, respectively. Since the functions $\ln h_{1}(p)$ and $\ln \bar{h}_{1}(p)$ are not concave but convex, we cannot apply our result (Theorem 3.2) to these cases. However, Theorems 5.2 and 5.3 provide sufficient conditions for dominance of species 2 in systems (4.2) and (4.3), respectively. Therefore, our results do not dominate the earlier ones by Franke and Yakubu [5, 6, 7], but have some advantages.

\section{REFERENCES}

[1] Chan, D. M. and Franke, J. E., Multiple extinctions in a discrete competitive system. Nonlinear Anal. Real World Appl. 2 (2001), 75-91. MR.1809865 (2001i:92039)

[2] de Feo, O. and Ferriere, R., Bifurcation analysis of population invasion: on-off intermittency and basin riddling, Internat. J. Bifur. Chaos Appl. Sci. Engrg. 10 (2000), 443-452. MR.1754082

[3] Elaydi, S. and Yakubu, A.-A., Global stability of cycles: Lotka-Volterra competition model with stocking, J. Difference Equ. Appl. 8 (2002) 537-549. MR1903949 (2004a:92031)

[4] Franke, J. E. and Yakubu, A.-A., Global attractors in competitive systems, Nonlinear Anal. 16 (1991), 111-129. MR1090785 (92a:58087)

[5] Franke, J. E. and Yakubu, A.-A., Mutual exclusion versus coexistence for discrete competitive systems, J. Math. Biol. 30 (1991), 161-168. MR1138846|(93a:92010)

[6] Franke, J. E. and Yakubu, A.-A., Geometry of exclusion principles in discrete systems, J. Math. Anal. Appl. 168 (1992), 385-400. MR.1175998 (93g:39002)

[7] Franke, J. E. and Yakubu, A.-A., Species extinction using geometry of level surfaces, Nonlinear Anal. 21 (1993), 369-378. MR.1237128 (94m:92008)

[8] Geritz, S. A. H., Gyllenberg, M., Jacobs, F. J. A. and Parvinen, K., Invasion dynamics and attractor inheritance, J. Math. Biol. 44 (2002), 548-560. MR1917846 (2003d:92021)

[9] Hofbauer, J., Hutson, V. and Jansen, W., Coexistence for systems governed by difference equations of Lotka-Volterra type, J. Math. Biol. 25 (1987), 553-570. MR0915090(89a:92059)

[10] Hofbauer, J., A unified approach to persistence, Acta Appl. Math. 14 (1989), 11-22. MR0990032(90e:92064)

[11] Hutson, V., Moran, W. and Vickers, G. T., On a criterion for survival of species in models governed by difference equations, J. Math. Biol. 18 (1983), 89-90.

[12] Hutson, V., A theorem on average Liapunov functions, Monatsh. Math. 98 (1984) 267-275. MR0776353 (86c:34086)

[13] Kon, R. and Takeuchi, Y., Permanence of host-parasitoid systems, Nonlinear Anal. 47 (2001), 1383-1393. MR.1970745 (2004c:37215)

[14] Kon, R. and Takeuchi, Y., Permanence of 2-host 1-parasitoid systems, Dyn. Contin. Discrete Impuls. Syst. Ser. B Appl. Algorithms 10 (2003), 389-402. MR1973439(2004b:92027)

[15] Kon, R., Permanence of discrete-time Kolmogorov systems for two species and saturated fixed points, J. Math. Biol. 48 (2004), 57-81. MR2035520(2005b:37210)

[16] Ranta, E., Kaitala, V., Alaja, S. and Tesar, D., Nonlinear dynamics and the evolution of semeloparous and iteroparous reproductive strategies, Amer. Natur. 155 (2000), 294-300.

[17] Yakubu, A.-A., The effects of planting and harvesting on endangered species in discrete competitive systems, Math. Biosci. 126 (1995), 369-378. MR 1317925

Faculty of Mathematics, Kyushu University, Hakozaki 6-10-1, Higashiku, Fukuoka 812-8581, JAPAN

E-mail address: kon-r@math.kyushu-u.ac.jp 